Neutron spin-flip scattering of nanocrystalline cobalt

This article has been downloaded from IOPscience. Please scroll down to see the full text article.

2011 J. Phys.: Condens. Matter 23016003

(http://iopscience.iop.org/0953-8984/23/1/016003)

View the table of contents for this issue, or go to the journal homepage for more

Download details:

IP Address: 158.64.77.122

The article was downloaded on 07/12/2010 at 08:21

Please note that terms and conditions apply. 


\title{
Neutron spin-flip scattering of nanocrystalline cobalt
}

\author{
D Honecker ${ }^{1}$, F Döbrich ${ }^{1}$, C D Dewhurst ${ }^{2}$, A Wiedenmann ${ }^{2}$ and \\ A Michels ${ }^{1}$ \\ ${ }^{1}$ Laboratory for the Physics of Advanced Materials, University of Luxembourg, 162A Avenue \\ de la Faïencerie, L-1511 Luxembourg, Luxembourg \\ ${ }^{2}$ Institut Laue-Langevin, 6 Rue Jules Horowitz, BP 156, F-38042 Grenoble Cedex 9, France \\ E-mail: andreas.michels@uni.lu
}

Received 26 August 2010, in final form 18 October 2010

Published 6 December 2010

Online at stacks.iop.org/JPhysCM/23/016003

\begin{abstract}
We report results of longitudinal (one-dimensional) neutron polarization analysis on polycrystalline bulk Co with an average crystallite size of $D=10 \mathrm{~nm}$. The spin-flip small-angle neutron scattering (SANS) data are analyzed in the approach-to-saturation regime within the framework of micromagnetic theory. In particular, we provide a closed-form expression for the spin-flip SANS cross section $\mathrm{d} \Sigma^{ \pm \mp} / \mathrm{d} \Omega$. From the data analysis, we find a room-temperature value of $A=(2.6 \pm 0.1) \times 10^{-11} \mathrm{~J} \mathrm{~m}^{-1}$ for the exchange-stiffness constant, which agrees well with earlier data.

(Some figures in this article are in colour only in the electronic version)
\end{abstract}

\section{Introduction}

The downscaling of the individual building blocks of solidstate materials to the nanometer scale results in an increase of the interface-to-volume ratio and in concomitant structural disorder due to the local misfit of the building units [1]. For nanocrystalline magnetic bulk materials, the structural disorder due to the relatively large volume fraction of internal interfaces (grain or phase boundaries) is accompanied by disorder in the magnetic microstructure, which is a consequence of the existence of the magnetoelastic coupling energy that links the strain tensor to the magnetization vector field [2]. On top of interface-induced spin disorder, the randomly fluctuating magnetic anisotropy field of the individual nanocrystallites may represent an additional source of spin nonuniformity [3].

Polarized neutron scattering [4] is an ideal technique for studying these types of spin disorder, since it is a bulk probe with a spatial resolution that extends from the unitcell dimension to the micron range. In particular, neutron polarization analysis (e.g. [5-12]) allows for the separation of nuclear and magnetic scattering, and recent progress in the development of ${ }^{3} \mathrm{He}$ spin filters [13] enables one to investigate magnetic nanostructures by means of longitudinal polarization analysis on a small-angle neutron scattering
(SANS) instrument [14-16]. ${ }^{3}$ As is well known, the interesting spin-misalignment scattering can be ideally studied in the spinflip channel (see below), which does not contain the nuclear coherent scattering.

In this article, we report our recent polarized neutron results on polycrystalline Co with an average crystallite size of $10 \mathrm{~nm}$. This material may be taken as a prototypical nanocrystalline random anisotropy ferromagnet. The spin-flip data are analyzed within a recent theoretical description of magnetic SANS of random anisotropy ferromagnets based on the continuum theory of micromagnetics [17].

\section{Experimental details}

The neutron experiments were carried out at room temperature at the SANS instrument D22 at the Institut Laue-Langevin, Grenoble, France [18]. The mean wavelength of the incident neutron beam was $\lambda=8 \AA$ with a wavelength spread of $\Delta \lambda / \lambda=10 \%$ (FWHM). A remnant FeSi supermirror transmission polarizer was used to select one of the two neutron-spin states. The incident neutron polarization was reversed by means of an rf spin flipper. Measurement of the four partial neutron intensities $I^{++}, I^{--}, I^{+-}$, and $I^{-+}$

3 Note that instead of longitudinal or one-dimensional polarization analysis, the term uniaxial polarization analysis is also found in the literature. 
became possible through a polarized ${ }^{3} \mathrm{He}$ spin-filter cell, which was placed inside a homogeneous-field cavity. The initial polarization of the ${ }^{3} \mathrm{He}$ nuclear magnetic moments is about $70 \%$, and their relaxation time is typically 200$300 \mathrm{~h}$ [13]. Magnetic guide fields of the order of $1 \mathrm{mT}$ served to maintain the polarization between polarizer, rf flipper, and ${ }^{3} \mathrm{He}$ filter. An electromagnet provided the external magnetic field perpendicular to the incoming neutron beam. The scattered neutrons were detected by a multi-tube detector which consists of 128 pixels $\times 128$ pixels (resolution: $8 \mathrm{~mm} \times 8 \mathrm{~mm}$ ). Rawdata treatment was carried out by means of the GRASP [18] and BerSANS [14, 19] software packages. Due to the limited beamtime and in the interest of exploring a maximum range within the experimental parameter space, we have not performed a so-called spin-leakage correction. Based on the measurement of the flipping ratio, we estimate that at most about $10-15 \%$ of 'spin contamination' (from the two non-spinflip channels) may be contained in the present spin-flip (sf) data.

The nanocrystalline Co sample was prepared by pulsed electrodeposition. The average crystallite size of the Co specimen was determined by analysis of wide-angle x-ray diffraction data and found to be $D=9.5 \pm 3.0 \mathrm{~nm}$. For more details on sample characterization, see [20].

\section{The spin-flip SANS cross section}

For the scattering geometry where the internal magnetic field $\mathbf{H}_{\mathrm{i}} \| \mathbf{e}_{z}$ is normal to the wavevector $\mathbf{k}_{0} \| \mathbf{e}_{x}$ of the incident neutron beam, the scattering vector $\mathbf{q}$ varies in the $\mathbf{e}_{y}-\mathbf{e}_{z}$ plane, and the angle $\theta$ is used to specify the orientation between $\mathbf{H}_{\mathrm{i}}$ and $\mathbf{q}$, i.e., $\mathbf{q} \cong q(0, \sin \theta, \cos \theta)$. The elastic differential spin-flip (sf) cross sections of an isotropic polycrystalline ferromagnet, $\mathrm{d} \Sigma^{ \pm \mp} / \mathrm{d} \Omega$, can be written as $[10,15-17,21,22]$

$$
\begin{aligned}
& \frac{\mathrm{d} \Sigma^{ \pm \mp}}{\mathrm{d} \Omega}(\mathbf{q})=\frac{8 \pi^{3}}{V} b_{\mathrm{H}}^{2}\left(\left|\tilde{M}_{x}\right|^{2}+\left|\tilde{M}_{y}\right|^{2} \cos ^{4} \theta\right. \\
& \left.\quad+\left|\tilde{M}_{z}\right|^{2} \sin ^{2} \theta \cos ^{2} \theta\right),
\end{aligned}
$$

where $V$ denotes the scattering volume, $\tilde{\mathbf{M}}(\mathbf{q})=\left[\tilde{M}_{x}(\mathbf{q})\right.$, $\left.\tilde{M}_{y}(\mathbf{q}), \tilde{M}_{z}(\mathbf{q})\right]$ represents the Fourier transform of the magnetization vector field $\mathbf{M}(\mathbf{x})=\left[M_{x}(\mathbf{x}), M_{y}(\mathbf{x}), M_{z}(\mathbf{x})\right]$, and $b_{\mathrm{H}}=2.7 \times 10^{-15} \mathrm{~m} / \mu_{\mathrm{B}}$, where $\mu_{\mathrm{B}}$ is the Bohr magneton. For polycrystalline magnetic materials, where helical scattering terms are of no relevance, the two sf cross sections are independent of the polarization of the incident neutrons and are expected to be identical (see, e.g., figure 4 in [15]). In writing down (1), we have neglected a cross term $\frac{\mathrm{d} \Sigma^{ \pm \mp}}{\mathrm{d} \Omega} \propto \tilde{M}_{y} \tilde{M}_{z} \sin \theta \cos ^{3} \theta[15,16]$, which is expected to cancel out for isotropic polycrystalline ferromagnets.

The information on the underlying magnetic microstructure in (1) is contained in the functions $\tilde{\mathbf{M}}(\mathbf{q})$, which depend on the magnetic-interaction parameters and on the applied magnetic field. As outlined in [17], by means of micromagnetic theory $[2,23-26]$ it becomes possible to derive a closed-form expression for the magnetization vector field for the particular case that the magnetic moments deviate only slightly from the applied-field direction. For $q_{\mathrm{x}}=$ 0 (as adapted to the above described scattering geometry), the small-misalignment solution of Brown's equations for the Fourier coefficient $\tilde{\mathbf{M}}(\mathbf{q})=\left(\tilde{M}_{x}, \tilde{M}_{y}, 0\right)$ of the transversal magnetization can be expressed as [17]

$$
\begin{gathered}
\tilde{M}_{x}(\mathbf{q})=\frac{M_{\mathrm{s}} h_{x}(\mathbf{q})}{H_{\mathrm{eff}}\left(q, H_{\mathrm{i}}\right)}, \\
\tilde{M}_{y}(\mathbf{q})=\frac{M_{\mathrm{s}} h_{y}(\mathbf{q})}{H_{\mathrm{eff}}\left(q, H_{\mathrm{i}}\right)+M_{\mathrm{s}} \sin ^{2} \theta},
\end{gathered}
$$

where $h_{x}(\mathbf{q})$ and $h_{y}(\mathbf{q})$ represent the Cartesian components of the Fourier transform of the perturbing anisotropy field $\mathbf{H}_{\mathrm{p}}(\mathbf{x})$, and $H_{\mathrm{eff}}\left(q, H_{\mathrm{i}}\right)=H_{\mathrm{i}}\left(1+l_{\mathrm{H}}^{2} q^{2}\right)$ and $l_{\mathrm{H}}\left(H_{\mathrm{i}}\right)=\sqrt{2 A /\left(\mu_{0} M_{\mathrm{s}} H_{\mathrm{i}}\right)}$ denote, respectively, the effective magnetic field and the exchange length of the field $\left(M_{\mathrm{s}}\right.$ : saturation magnetization; $A$ : exchange-stiffness parameter; $\mu_{0}$ : permeability of free space).

By inserting (2) in (1) and following the arguments in [17], we can express the sf cross section of an isotropic polycrystalline ferromagnet near saturation as

$$
\begin{gathered}
\frac{\mathrm{d} \Sigma^{ \pm \mp}}{\mathrm{d} \Omega}\left(q, H_{\mathrm{i}}, \theta\right)=S_{\mathrm{H}}(\mathbf{q}) R\left(q, H_{\mathrm{i}}, \theta\right) \\
+\frac{8 \pi^{3}}{V} b_{\mathrm{H}}^{2}\left|\tilde{M}_{z}\right|^{2} \sin ^{2} \theta \cos ^{2} \theta,
\end{gathered}
$$

where $S_{\mathrm{H}}(\mathbf{q})=8 \pi^{3} V^{-1} b_{\mathrm{m}}^{2} \rho_{\mathrm{a}}^{2} M_{\mathrm{s}}^{-2} h^{2}(\mathbf{q})$ is the so-called anisotropy-field scattering function, and $h^{2}(\mathbf{q})=h_{x}^{2}(\mathbf{q})+$ $h_{y}^{2}(\mathbf{q})\left(b_{\mathrm{m}}\right.$ : atomic magnetic scattering length; $\rho_{\mathrm{a}}$ : atomic density). The dimensionless micromagnetic response function for SANS takes on the form

$$
R\left(q, H_{\mathrm{i}}, \theta\right)=\frac{p^{2}}{2}\left(1+\frac{\cos ^{4} \theta}{\left(1+p \sin ^{2} \theta\right)^{2}}\right),
$$

where $p\left(q, H_{\mathrm{i}}\right)=M_{\mathrm{s}} / H_{\text {eff. }}$ These equations predict enhanced scattering along the field direction, since $R\left(\theta=0^{\circ}\right)=p^{2}$ and $R\left(\theta=90^{\circ}\right)=p^{2} / 2$. If the a priori unknown real functions $\tilde{M}_{z}(\mathbf{q})$ and $S_{\mathrm{H}}(\mathbf{q})$ depend only on the magnitude $q$ of the scattering vector, one can perform the average of (3) with respect to the angle $\theta$, i.e., $(2 \pi)^{-1} \int_{0}^{2 \pi}(\cdots) \mathrm{d} \theta$, to obtain

$$
\frac{\mathrm{d} \Sigma^{ \pm \mp}}{\mathrm{d} \Omega}\left(q, H_{\mathrm{i}}\right)=S_{\mathrm{H}}(q) R\left(q, H_{\mathrm{i}}\right)+f(q)
$$

where

$$
R\left(q, H_{\mathrm{i}}\right)=\frac{1}{2}+\frac{p^{2}}{2}-\frac{1}{4}(2-p) \sqrt{1+p},
$$

and $f(q)=\pi^{3} V^{-1} b_{\mathrm{H}}^{2} \tilde{M}_{z}^{2}(q)$. As a consequence of the factor $\cos ^{4} \theta$ in (4), rather than $\cos ^{2} \theta$, the micromagnetic response function for sf scattering is slightly different from the $R$ for the case of unpolarized neutrons, which reads $R=p^{2} / 2+$ $p^{2} /(4 \sqrt{1+p})[17]$.

The numerical value of the response function is known for given materials parameters $A$ and $M_{\mathrm{s}}$ and at each experimental $q$ and $H_{\mathrm{i}}$ value. Hence, from straight-line fits of plots of the measured $\mathrm{d} \Sigma^{ \pm \mp} / \mathrm{d} \Omega$ versus $R$ at discrete $q=q^{\prime}$ and for various $H_{\mathrm{i}}$, one can determine $S_{\mathrm{H}}$ (slope) and $f$ (intercept) at the particular $q^{\prime}$ value (compare (5) and figure 7 in [17]); note that we neglect the field dependence of $\tilde{M}_{z}^{2}(q)$, which is justified in the approach-to-saturation regime. 

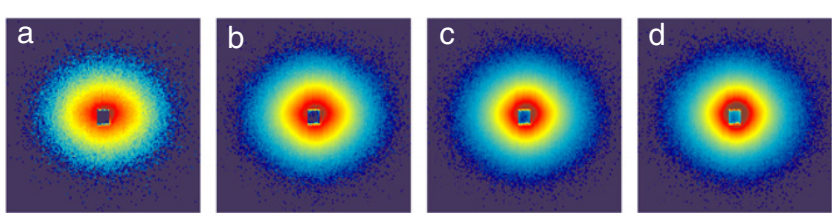

Figure 1. Spin-flip scattering cross section of nanocrystalline Co on the 2D area detector at various internal magnetic fields $H_{\mathrm{i}}$ (horizontal): (a) $1239 \mathrm{mT}$; (b) $181 \mathrm{mT}$; (c) $53 \mathrm{mT}$; (d) $24 \mathrm{mT}$. The $q$ range (from the center to the corner of the detector) corresponds to $0.03 \mathrm{~nm}^{-1} \lesssim q \lesssim 0.5 \mathrm{~nm}^{-1}$.

However, compared to the case of unpolarized neutrons, where the intercept value that is obtained from such a data analysis contains also the nuclear scattering, the function $f(q)$ exclusively provides information on the residual magnetic scattering due to fluctuations in the longitudinal magnetization Fourier component $\tilde{M}_{z}^{2}$. In other words, $f(q)$ describes the magnetic scattering which is not related to the micromagnetic structures under study.

For the analysis of the 2D magnetic SANS data (see figure 1), we have computed radial (azimuthal) averages of the anisotropic data. This procedure allows for a convenient comparison with the theoretical expression (5) for the radially averaged spin-misalignment SANS cross section.

In order to test these theoretical predictions, we discuss in the following sf data of nanocrystalline Co.

\section{Experimental results and discussion}

Figure 1 illustrates the field dependence of the sf cross section $\mathrm{d} \Sigma^{ \pm} / \mathrm{d} \Omega$ of nanocrystalline Co in the $2 \mathrm{D}$ detector plane, and figure 2 depicts the corresponding radially averaged data. For all fields investigated, $\mathrm{d} \Sigma^{ \pm} / \mathrm{d} \Omega$ exhibits enhanced scattering along the field direction, and the radially averaged $\mathrm{d} \Sigma^{ \pm} / \mathrm{d} \Omega$ decreases by more than two orders of magnitude as $H_{\mathrm{i}}$ increases from 24 to $1239 \mathrm{mT}$. This observation clearly demonstrates the existence of strong spin-misalignment scattering from transversal magnetization components. The solid lines in figure 2 represent the prediction of the micromagnetic theory, equation (5). Note that applied fields of the order of a few $10 \mathrm{mT}$ are required for approaching saturation (compare figure 4 in [20]). From these fits we obtain $A=(2.6 \pm 0.1) \times 10^{-11} \mathrm{~J} \mathrm{~m}^{-1}$ for the exchangestiffness constant, a value which agrees within error bars with earlier data for this quantity obtained by analysis of unpolarized SANS data [20]. For comparison, we also quote the corresponding value for the spin-wave stiffness constant, $D=460 \pm 20 \mathrm{meV} \AA^{2}$, where $D$ was computed according to $D=2 A g \mu_{\mathrm{B}} / M_{\mathrm{s}}$ using the $g$-factor of Co, $g=2.21$, and $M_{\mathrm{s}}=1434 \mathrm{kA} \mathrm{m}^{-1}$ [20]. This value for $D$ agrees well with literature data obtained on a Co single crystal by inelastic neutron scattering [27].

Also shown in figure 2 are the anisotropy-field scattering function $S_{\mathrm{H}}(q)$ and $f(q)$. While $S_{\mathrm{H}}(q)$ reflects the spatial structure of the magnetic anisotropy field, $f(q)$ is directly proportional to the magnitude-square of the Fourier coefficient of the longitudinal magnetization component $\tilde{M}_{z}^{2}(q)$. It

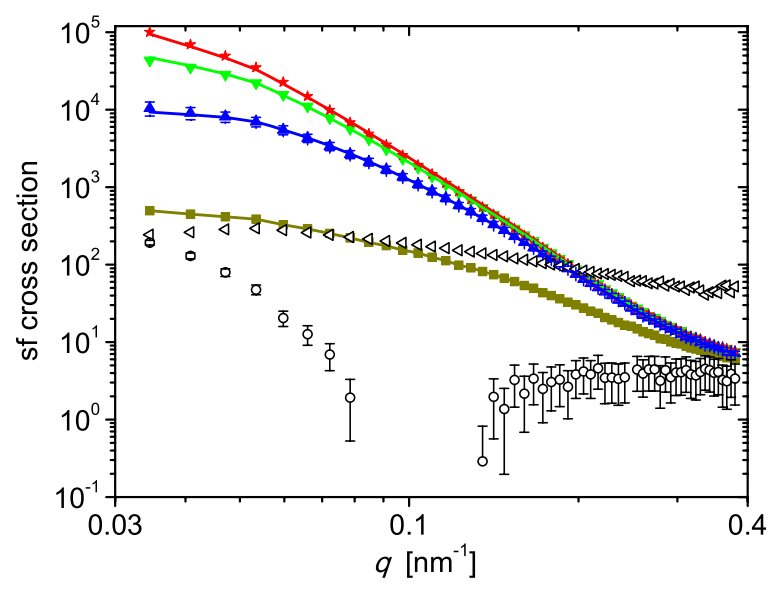

Figure 2. Field dependence of the radially averaged sf scattering of nanocrystalline Co. Field values (from top to bottom): ( $\star$ ) $24 \mathrm{mT}$;

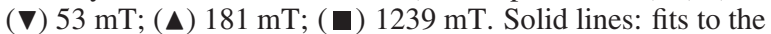
micromagnetic model, equation (5). The open symbols (O) and $(\triangleleft)$ represent, respectively, the function $f(q) \propto \tilde{M}_{z}^{2}(q)$ and the anisotropy-field scattering function $S_{\mathrm{H}}(q)$.

is readily seen that the total sf scattering is significantly exceeding this contribution at all experimental $q$ and even at the highest field of $1239 \mathrm{mT}$. This suggests that the residual small-angle scattering due to fluctuations in the longitudinal magnetization is small relative to the spin-misalignment scattering. In other words, spatial variations in the magnitude of the magnetization, $M_{\mathrm{s}}(\mathbf{x})$, play only a minor role for the magnetic SANS of nanocrystalline electrodeposited Co, a conclusion which is in accordance with the micromagnetic model where uniform values for $A$ and $M_{\mathrm{s}}$ are assumed.

Analysis of $S_{\mathrm{H}}(q)$ provides lower bounds for the magnitude of the mean anisotropy and magnetostatic field [28]. For instance, the mean-square anisotropy field $\left\langle\left|\mathbf{H}_{\mathrm{p}}\right|^{2}\right\rangle_{v}$ can be estimated according to $\left\langle\left|\mathbf{H}_{\mathrm{p}}\right|^{2}\right\rangle_{v} \propto \int_{q_{\min }}^{q_{\max }} S_{\mathrm{H}}(q) q^{2} \mathrm{~d} q$, where $q_{\min }$ and $q_{\max }$ refer, respectively, to the lower and upper limit of experimental scattering vectors. (A similar formula can be used to determine the mean magnetostatic field.) However, such an analysis could not be carried out here, since the respective integrands did not show signs of convergence within $0.03 \mathrm{~nm}^{-1} \lesssim q \lesssim 0.4 \mathrm{~nm}^{-1}$ (roughly $S_{\mathrm{H}}(q) \propto q^{-1}$ ), suggesting that there are significant scattering contributions outside this range.

Equation (4) predicts $R\left(q, H_{\mathrm{i}}, \theta=0^{\circ}\right) / R\left(q, H_{\mathrm{i}}, \theta=\right.$ $\left.90^{\circ}\right)=2$. In order to illustrate the field dependence of the azimuthal anisotropy of the scattering pattern on the detector, figure 3 shows the ratio $r\left(H_{\mathrm{i}}\right)$ of $\pm 7.5^{\circ}$ sector averages of the scattering parallel over normal to the applied-field direction. The data exhibit $r \leqslant 2$, in reasonable agreement with (3). Note that the value of $r=2$ is expected to be strictly valid only for $\theta=0^{\circ}$ and for $90^{\circ}$; sector averaging and additional scattering contributions due to spin leakage may result in $r<2$.

\section{Summary and conclusions}

By means of longitudinal (one-dimensional) neutron polarization analysis, we have measured the spin-flip (sf) smallangle neutron scattering (SANS) cross section $\mathrm{d} \Sigma^{ \pm \mp} / \mathrm{d} \Omega$ of 


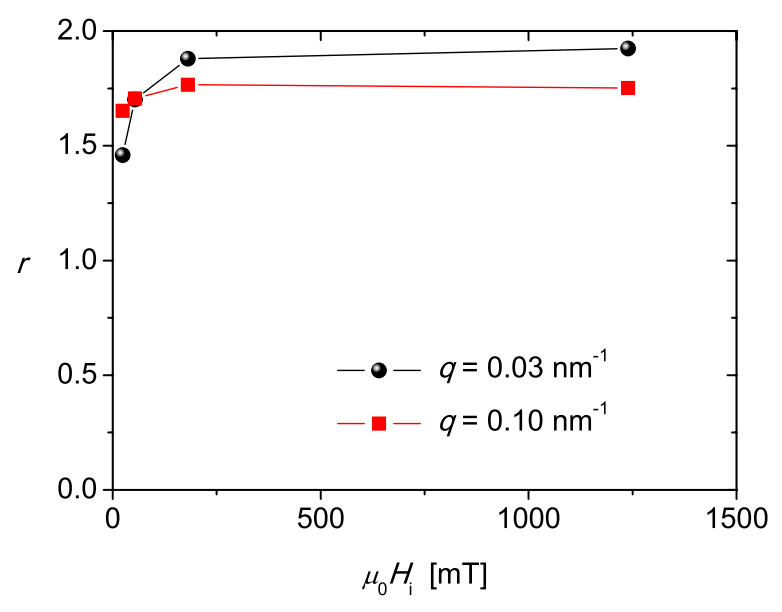

Figure 3. Field dependence of the ratio $r$ of the sf scattering $\left( \pm 7.5^{\circ}\right.$ sector averages) parallel $\left(\theta=0^{\circ}\right)$ over perpendicular $\left(\theta=90^{\circ}\right)$ to the applied-field direction at selected $q$ values (see inset). The lines are to guide the eyes.

nanocrystalline bulk Co with an average crystallite size of $D=10 \mathrm{~nm}$. Using linearized micromagnetic theory, we have derived a closed-form expression for $\mathrm{d} \Sigma^{ \pm \mp} / \mathrm{d} \Omega$. We find that the variation of the radially averaged $\mathrm{d} \Sigma^{ \pm \mp} / \mathrm{d} \Omega$ with the scattering vector and the applied magnetic field can be excellently reproduced by the theory. From the analysis, a value of $A=(2.6 \pm 0.1) \times 10^{-11} \mathrm{~J} \mathrm{~m}^{-1}$ for the exchangestiffness constant is determined, which is in good agreement with published data determined by unpolarized SANS and by inelastic neutron scattering on single crystals. In contrast to the case of unpolarized SANS, the present approach provides the magnitude-square of the Fourier coefficient of the longitudinal magnetization component $\tilde{M}_{z}^{2}(q)$. It is concluded that the experimental data for nanocrystalline Co support well the predictions of the micromagnetic model.

\section{Acknowledgments}

This study was financially supported by the Institut LaueLangevin, by the Deutsche Forschungsgemeinschaft (Grants No. MI 738/3-2 and No. MI 738/6-1) and by the National Research Fund of Luxembourg in the framework of the ATTRACT project FNR/A09/01. The nanocrystalline Co sample was kindly provided by Professor Uwe Erb from the University of Toronto.

\section{References}

[1] Skomski R 2003 J. Phys.: Condens. Matter 15 R841

[2] Kronmüller H and Fähnle M 2003 Micromagnetism and the Microstructure of Ferromagnetic Solids (Cambridge: Cambridge University Press)

[3] Döbrich F, Elmas M, Ferdinand A, Markmann J, Sharp M, Eckerlebe H, Kohlbrecher J, Birringer R and Michels A 2009 J. Phys.: Condens. Matter 21156003

[4] Halpern O and Johnson M H 1939 Phys. Rev. 55898

[5] Sáenz A W 1960 Phys. Rev. 1191542

[6] Maleyev S V 1961 Sov. Phys. JETP 13860

[7] Izyumov Yu A and Maleyev S V 1962 Sov. Phys. JETP 141168

[8] Maleyev S V, Baryakhtar V G and Suris R A 1963 Sov. Phys.-Solid State 42533

[9] Blume M 1963 Phys. Rev. 1301670

[10] Moon R M, Riste T and Koehler W C 1969 Phys. Rev. 181920

[11] Okorokov A I, Runov V V and Gukasov A G 1978 Nucl. Instrum. Methods 157487

[12] Tasset F 1989 Physica B 156-157 627

[13] Petoukhov A K, Guillard V, Andersen K H, Bourgeat-Lami E, Chung R, Humblot H, Jullien D, Lelièvre-Berna E, Soldner T, Tasset F and Thomas M 2006 Nucl. Instrum. Methods A $\mathbf{5 6 0} 480$

[14] Keiderling U, Wiedenmann A, Rupp A, Klenke J and Heil W 2008 Meas. Sci. Technol. 19034009

[15] Honecker D, Ferdinand A, Döbrich F, Dewhurst C D, Wiedenmann A, Gómez-Polo C, Suzuki K and Michels A 2010 Eur. Phys. J. B 76 209-13

[16] Krycka K L, Booth R A, Hogg C R, Ijiri Y, Borchers J A, Chen W C, Watson S M, Laver M, Gentile T R, Dedon L R, Harris S, Rhyne J J and Majetich S A 2010 Phys. Rev. Lett. 104207203

[17] Michels A and Weissmüller J 2008 Rep. Prog. Phys. 71066501

[18] For more details, see, http://www.ill.eu/d22

[19] Keiderling U 2002 Appl. Phys. A 74 S1455

[20] Weissmüller J, Michels A, Barker J G, Wiedenmann A, Erb U and Shull R D 2001 Phys. Rev. B 63214414

[21] Schärpf O and Capellmann H 1993 Phys. Status Solidi a 135359

[22] Schweizer J 2006 Neutron Scattering from Magnetic Materials ed T Chatterji (Amsterdam: Elsevier) pp 153-213

[23] Brown W F Jr 1963 Micromagnetics (New York: Interscience)

[24] Shtrikman S and Treves D 1963 Magnetism III vol 3, ed G T Rado and H Suhl (New York: Academic) pp 395-414

[25] Aharoni A 1996 Introduction to the Theory of Ferromagnetism 2nd edn (Oxford: Clarendon)

[26] See, e.g. Kronmüller H and Parkin S (ed) 2007 Handbook of Magnetism and Advanced Magnetic Materials vol 2 Micromagnetism (Chichester: Wiley)

[27] Alperin H A, Steinsvoll O, Shirane G and Nathans R 1966 J. Appl. Phys. 371052

[28] Michels A, Weissmüller J and Birringer R 2002 Eur. Phys. J. B 29533 